\title{
Pain Reduction and Improvement in Range of Motion After Daily Consumption of an Açai (Euterpe oleracea Mart.) Pulp-Fortified Polyphenolic-Rich Fruit and Berry Juice Blend
}

\author{
Gitte S. Jensen, David M. Ager, Kimberlee A. Redman, ${ }^{1}$ Marcie A. Mitzner, \\ Kathleen F. Benson, and Alexander G. Schauss ${ }^{3}$ \\ ${ }^{1}$ NIS Labs, Klamath Falls, Oregon, USA. \\ ${ }^{2}$ Cascade Chiropractic and Rehabilitation, Klamath Falls, Oregon, USA. \\ ${ }^{3}$ Natural and Medicinal Products Research, AIBMR Life Sciences, Puyallup, Washington, USA.
}

\begin{abstract}
Dietary interventions involving antioxidants are of interest for reducing inflammation, improving joint motion, and altering pain perception. We evaluated the effect of oral consumption of a fruit and berry blend on pain and range of motion (ROM). This open-label clinical pilot study involved 14 study participants with limitations in ROM that was associated with pain and affected daily living. Participants included but were not limited to those with age-related osteoarthritis. Study participants consumed $120 \mathrm{~mL}$ MonaVie Active ${ }^{\circledR}$ fruit juice, predominantly containing açai pulp (Euterpe oleracea Mart.) and other fruit concentrates, daily for 12 weeks. Study participants were assessed at baseline and 2, 4, 8, and 12 weeks by structured nurse interviews, pain and activities of daily living (ADL) questionnaires, blood samples, and ROM assessment. Pain was scored by using a visual analogue scale. ROM was assessed by using dual digital inclinometry as recommended by American Medical Association guidelines. Consumption of the juice resulted in significant pain reduction, improved ROM measures, and improvement in ADLs. Serum antioxidant status, as monitored by the cell-based antioxidant protection in erythrocytes (CAP-e) assay, was improved within 2 weeks and continued to improve throughout the 12 weeks of study participation $(P<.01)$. The inflammatory marker $\mathrm{C}$-reactive protein was reduced at 12 weeks, but this change did not reach statistical significance. Lipid peroxidation decreased mildly at 12 weeks. The antioxidant status, as measured by the CAP-e bioassay, showed the best correlation with improvements in physical well-being (pain, ROM, and ADL). The significant association among increased antioxidant status, improved ROM, and pain reduction warrants further study.
\end{abstract}

KEY WORDS: $\bullet C A P-e \bullet$ range of motion $\bullet$ digital inclinometry $\bullet$ pain $\bullet$ visual analogue scale $\bullet$ inflammation $\bullet$ adults - geriatrics • antioxidants • polyphenols • açai • Euterpe oleracea

\section{INTRODUCTION}

$\mathbf{T}$ HE DECLINE IN FUNCTION associated with normal aging closely mimics the effects of immobilization and inactivity. The ability to maintain daily exercise involving moderate physical activity can dramatically slow the decline in function. ${ }^{1,2}$ The importance of maintaining mobility is illustrated by a decade-long prospective study of the elderly that found a decline in maximal oxygen consumption of $12 \%$ in 1 decade compared with age-matched runners, who experienced only a $5.5 \%$ decline. ${ }^{3}$ Maintaining higher levels of flexibility, mobility, muscle strength, and aerobic capacity not only contributes to healthy aging but also preserves independent functioning of daily activities.

Several mechanisms are involved, and each may be supported by dietary components for healthier aging with a

Manuscript received 13 June 2010. Revision accepted 5 October 2010.

Address correspondence to: Gitte S. Jensen, Holger NIS Labs, 1437 Esplanade Avenue, Klamath Falls, OR 97601, USA, E-mail: gitte@ nislabs.com better quality of life. These mechanisms include antioxidant and anti-inflammatory support, pain reduction by analgesic effects, and direct effects on gene expression. Berry polyphenols have been implemented to affect all 3 mechanisms, ${ }^{4-7}$ and consumption of certain berries therefore receives special attention as dietary support for healthy aging.

The Amazonian fruit açai (Euterpe oleracea Mart.) has been studied extensively for its nutritional and phytochemical composition and been found to contain compounds with potent anti-inflammatory ${ }^{8-10}$ and antioxidant properties. ${ }^{11}$ Recent bioactivity studies of the compounds in açai pulp have revealed a group of polyphenolic flavonoids that could enter the cytosol and reduce oxidative damage associated with inflammation within the cell. ${ }^{9,10}$ Because açai-rich juice contains a range of fruits and berries, with açai pulp the predominant ingredient, it would be rich in flavonoids with strong antioxidant activities and anti-inflammatory properties. This was confirmed by purifying compounds in the pulp and the juice and demonstrating that specific polyphenol 
compounds trigger a reduction in the production of reactive oxygen species and exhibit potent anti-inflammatory effects seen at even the lowest dose tested. ${ }^{12}$ Chrysoerial, a flavone, purified from the pulp, exhibited these properties in human polymorphonuclear cells, along with many aglycone polyphenolics. Other isolated antioxidant compounds, including luteolin, quercetin, and dihydrokaempferol, had the capacity to enter live cells and protect them from oxidative damage by using the cell-based antioxidant protection in erythrocytes (CAP-e) bioassay. ${ }^{12}$

The in vitro evidence for the potent antioxidant and antiinflammatory properties of açai pulp is further supported by studies in humans. ${ }^{13}$ Two studies, including a randomized, double-blind, placebo-controlled, crossover study, reported significant reductions in lipid peroxidation and increased free radical scavenging in vivo when healthy participants age 19-52 years were fed an açai pulp-rich juice. ${ }^{10}$ Consumption of a single acute dose of 4 ounces of an açai-rich juice resulted in a rapid increase in antioxidant activity in the serum, as measured by the CAP-e assay. This increased serum antioxidant activity translated into positive downstream consequences, as measured by a reduction in lipid peroxidation within 2 hours after consumption of the açai juice blend.

Pain is a sensory measure and is subjective in nature, but it has a molecular basis involving synaptic activity and release of neurotransmitters. When pain reduces mobility of critical joints, inflammatory molecules that mitigate the pain or initiate repair build up. Analgesic (pain-reducing) effects involve direct effects on signal transduction (nociception) in the nerve system, as well as cyclooxygenase-2 (COX-2) enzyme inhibitors that lead to reduced levels of prostaglandins; this results in a combined pain-reducing effect and an anti-inflammatory effect. Some berries contain antinociceptive compounds, which may be linked to COX-2 inhibition. ${ }^{4-7}$ Açai contains COX-2-inhibiting compounds, ${ }^{9}$ which may suggest a potential antinociceptive effect.

Direct effects of açai pulp as a food ingredient on expression of genes involved in pain, resistance to oxidative stress, and metabolism was studied in the fruit fly, Drosophila melanogaster. ${ }^{14-16}$ The life extension results from açai pulp were found to be due in part to activation of the c-Jun $\mathrm{N}$-terminal kinase signaling pathway, ${ }^{14}$ which is involved in many conditions, such as diabetes, neurodegeneration, liver disease, and pain. ${ }^{17}$

Given the combined antioxidant, anti-inflammatory, and potentially antinociceptive properties of açai juice and pulp seen in vitro and in vivo, we sought to conduct a pilot study in humans with chronic pain and underlying inflammatory issues. Subsequently, we reviewed numerous case reports on the ability of an unclarified/unfiltered juice enriched with the pulp of açai and fruit concentrates to improve range of motion (ROM) and reduce pain. The test product for this study was MonaVie Active ${ }^{\circledR}$ (MonaVie LLC, South Jordan, UT, USA), a fruit- and berry-based juice blend with a high level of polyphenolic compounds that exhibit strong antioxidant properties. These properties stem from the predominance of açai pulp in the formulation; pulp of this fruit has been shown to have high superoxide and peroxyl radical scavenging capacities in vitro. The juice has also been extensively studied for safety and thus is suitable for study in humans. ${ }^{18}$

This open-label pilot study of anti-inflammatory effects was conducted in older adults with some degree of chronic joint pain that affected ROM. The objective was to see whether consuming MonaVie Active twice daily over 12 weeks would affect ROM, perceived pain, antioxidant status, and inflammatory markers.

\section{MATERIALS AND METHODS}

\section{Study design}

Fourteen participants were enrolled in the 12-week study to identify the time course of changes in symptoms of pain and reduced ROM. Study participants underwent an intake interview; if they met the inclusion criteria they were recruited for study entry. A medical examination was performed to identify areas of primary symptoms, as well as to monitor severity of primary and secondary health concerns and pain. The medical examination also helped ensure that no other health problems would prevent any person from participating, such as a condition that would require intensive medical treatment. The study participants were monitored at baseline and after 2, 4, 8, and 12 weeks. Blood was taken at each visit, and a structured nurse interview specifically addressed any subjective changes. A medical examination performed at study exit ensured that no additional health conditions had developed during the study. Figure 1 summarizes the study design.

\section{Consumable}

The pilot study was conducted as an open-label study, so no placebo control was used. The MonaVie Active juice contains 8 fruits and berries that have been studied for their antioxidant properties (açai pulp, pomegranate, wolfberry, camu camu, passion fruit, aronia, acerola, and bilberry) and 10 common fruits (apricot, purple grape, white grape, lychee, banana, kiwi, pear, cranberry, blueberry, and prune). MonaVie Active also contains glucosamine hydrochloride, generally recognized as safe by the U.S. Food and Drug Administration as a functional food ingredient.

Each participant consumed approximately 1 bottle of MonaVie Active every 6 days for 12 weeks. Participants were given a measuring glass onto which the $2-\mathrm{oz}(60-\mathrm{mL})$

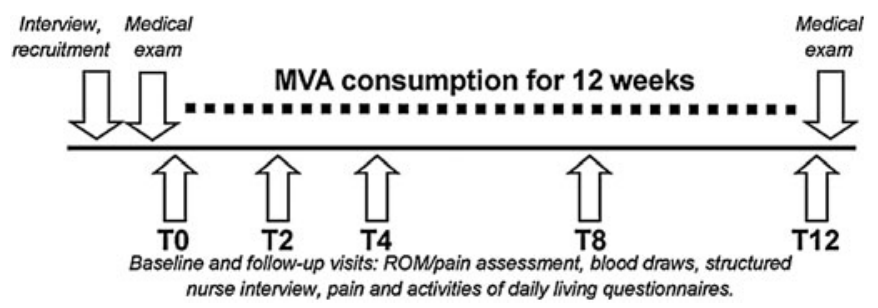

FIG 1. Study design for the 12-week study. Arrows indicate visits to the clinic, assessment of pain and range of motion (ROM), and blood draws. MVA, MonaVie Active 
level was etched, and instructed to consume 2 measures daily (equaling a daily dose of 4oz. [120 mL] of MonaVie Active per day).

\section{Study population}

The original plan was to involve 12 participants; however, during the initial recruitment and interview process 14 interested participants were identified. Because this study was performed with the intent of broadly exploring what pain conditions may benefit from consumption of MonaVie Active, we decided to enroll all 14 even though some did not meet the original inclusion criteria. Table 1 lists participant characteristics.

Inclusion criteria. Criteria for entry into the study were age 44-84 years, mild to moderate joint pain, and no other health problems.

Exclusion criteria. Patients were excluded if they met the following criteria: regular consumption of MonaVie Active or any similar antioxidant-rich juices or supplements during the 6 months before the beginning of the study, pregnancy, use of recreational drugs, history of chronic viral disease, and shellfish allergies. The last exclusion criterion was based on the content of glucosamine hydrochloride in MonaVie Active, which has since been changed to a plant-based source.

The originally proposed exclusion criteria were ongoing intensive medical treatment for other diseases (cancer, viral disease) and ongoing treatment with antidepressants or hypnotics. These were omitted after we chose to include a few participants with severe pain and fibromyalgia (which is often treated with antidepressants). The participant with the most severe condition had degenerative illness of the spine (ankylosing spondylitis) that required treatment with methadone.

\section{Method for assessing ROM}

ROM was evaluated comprehensively along the vertical weight-bearing axis of the body. Shoulder ROM was also assessed. Thus, the ROM evaluations not only focused on a person's primary area of concern but also evaluated changes in overall ROM. The rationale for this approach is that when a specific weight-bearing joint (such as left knee) is painful, other areas compensate and overall tension and ROM limitations build up.

The J-Tech Tracker Freedom system (JTech Medical, Salt Lake City, UT, USA) uses dual digital inclinometry protocols of the American Medical Association and wireless instruments to measure composite ROM and strength evaluation. This system is controlled with a foot switch; this allows all instrumented tests to be accurately recorded by the operator while probes are touching the study participant. The following instrumented tests were performed for this study: dual inclinometry (ROM) for spine and extremities, dynamometer muscle testing (strength), and grip testing (grip strength), all performed with the J-Tech Tracker Freedom. The assessment was performed by an Oregonlicensed chiropractor with 10 years of experience in ROM

Table 1. Study Sample, Areas of Pain, and Areas Improved During the 12-Week Study

\begin{tabular}{|c|c|c|c|c|c|c|c|}
\hline Volunteer $^{\mathrm{a}}$ & $\operatorname{Sex}$ & Age (y) & Primary complaint & Other pain & $\begin{array}{c}\text { ARROM } \\
\text { at study start }\end{array}$ & $\begin{array}{c}\text { ARROM } \\
\text { after } 12 \text { weeks }\end{array}$ & $A D L$ improvement $(\%)^{\mathrm{c}}$ \\
\hline 1 & M & 84 & Joint pain & Agent Orange rash & 19 & 10 & 22 \\
\hline 2 & $\mathrm{~F}$ & 68 & Joint pain & Right leg muscular & 14 & 7 & 32 \\
\hline 3 & $\mathrm{~F}$ & 47 & Joint pain (knees) & - & 8 & 4 & 30 \\
\hline 4 & $\mathrm{~F}$ & 59 & Rheumatoid arthritis & Right knee, hip & 17 & 8 & 20 \\
\hline 5 & M & 51 & Ankylosing spondylitis & Joint & 17 & 6 & \\
\hline 6 & $\mathrm{~F}$ & 44 & Joint pain (multiple) & - & 6 & 3 & -13 \\
\hline 7 & $\mathrm{~F}$ & 54 & Chronic muscle pain & Knee & 17 & 11 & 42 \\
\hline 8 & $\mathrm{~F}$ & 59 & Joint pain (knees) & - & 12 & 3 & -3 \\
\hline 9 & $\mathrm{~F}$ & 54 & Fibromyalgia & Lupus & 9 & 3 & \\
\hline 10 & $\mathrm{~F}$ & 70 & Joint pain & Lower back, hip/leg & 10 & 4 & -2 \\
\hline 11 & M & 47 & Joint pain & Knee and hip pain & 14 & 7 & 6 \\
\hline 12 & $\mathrm{~F}$ & 60 & Joint pain (knees) & Lower back pain & 6 & 2 & 19 \\
\hline 13 & M & 58 & Joint pain (knees) & Shoulder & 13 & 6 & -8 \\
\hline 14 & $\mathrm{~F}$ & 47 & Joint pain (knees) & Hand and finger & 7 & 3 & 11 \\
\hline Mean \pm SD & & $57.3 \pm 11.0$ & & & $12.1 \pm 4.5$ & $5.5 \pm 2.8$ & $13.0 \pm 17.3$ \\
\hline
\end{tabular}

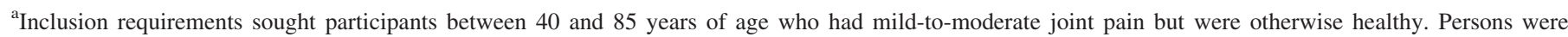
excluded if they reported regular consumption of MonaVie Active or similar antioxidant-rich juices or supplements during the 6 months before study entry, were pregnant, used recreational drugs, had a history of chronic viral disease, or had shellfish allergies. The actual study population had a diverse range of primary pain concerns, but joint pain was the most frequent.

${ }^{b}$ Areas of reduced range of motion were defined as joints with less than $75 \%$ of normal range of motion at study start. Twenty-eight areas of motion were evaluated by dual digital inclinometry. These areas were focused along the vertical weight-bearing column of the body and also included shoulder range of motion (see also Table 2).

'Activities of daily living questionnaire scores assessing a participant's ability to do various tasks (e.g., those involving reach, sitting, walking, climbing stairs, and transportation). The percentage improvement during the 12-week study is shown. Two patients were removed from the ADL analysis because they experienced greater pain than the rest of the study group: ankylosing spondylitis (volunteer 5) and fibromyalgia (volunteer 9).

ADL, activity of daily living; ARROM, area with reduced range of motion; F, female; M, male; SD, standard deviation. 
assessment. Thus, 1 operator performed all ROM assessments throughout the study.

These data were analyzed as follows: overall ROM for each anatomic area and specific assessment of each person's primary problem areas. The anatomic areas were cervical (neck) flexion, extension, left rotation, and right rotation; thoracic (upper body/torso) flexion and minimal kyphosis, left and right rotation; shoulder abduction, adduction, internal and external rotation; lumbar (lower back) flexion, extension, and lateral left and right motion; internal and external rotation of left and right hip, and left and right knee flexion/extension.

\section{Methods for assessing pain}

Five methods for pain assessments were used:

1. A substantial part of the structured nurse interview was focused on asking questions about pain and functionality. Changes in medication, particularly pain medication, were also tracked.

2. A visual analogue scale (VAS) was used to track current pain levels at each visit. The scale did not use increment marks and was $100 \mathrm{~mm}$ long. One end was labeled "no pain" and the other end was labeled "intense pain." The study participants were instructed to make a mark anywhere on the line reflecting their current pain level on the day of the visit. The score was measured on the scale in millimeters and was scored as a percentage.

3. A questionnaire focused on activities of daily living (ADLs) was administered. It addressed issues of functionality, such as problems walking on stairs, driving, dressing, and performing other tasks associated with daily life.

4. A questionnaire eliciting the nature of the pain was administered.

5. By using the J-Tech wireless equipment, which included an algometer, the 18 points associated with fibromyalgia and additional paraspinal points were tested. Pressure algometry uses a probe with a flat tip of a known surface area. It is pressed against the person's body at specific predetermined points. Increasing pressure is applied to the probe until the study participant reports discomfort. The probe electronically measures how many pounds of pressure were applied at the time the probe was removed from the body (upon the participant's report of pain). This testing is well documented as an objective component for evaluating point tenderness, particularly in chronic pain syndromes. This method is less associated with a person's ability to function and more associated with diagnostic measures of tissue pain empirically found to be involved in chronic pain and fatigue syndromes, probably because of inflammatory issues.

\section{Blood samples}

At each time point, 1 serum separator tube of peripheral venous blood was collected. The participants were in- structed not to consume MonaVie Active during the morning before a clinic visit to avoid any immediate short-term improvement seen in a previous study ${ }^{6}$ and allow tracking of long-term improvements. The coagulated blood samples in the serum separator tube vials were centrifuged for 10 minutes and the serum harvested and divided into aliquots in small freezer vials. These aliquots were frozen immediately and stored at $-80^{\circ} \mathrm{C}$ until tested. The serum samples were used for evaluating changes to the following measures: serum antioxidant testing using the CAP-e assay, serum lipid peroxidation, and high-sensitivity C-reactive protein (CRP).

\section{Reagents}

The following buffers and reagents were obtained from Sigma-Aldrich (St. Louis, MO, USA): gallic acid, Trolox, ascorbic acid, phosphate-buffered saline (PBS), RPMI-1640 culture medium, and hydrogen peroxide 30\% solution. Dichlorofluorescein diacetate (DCF-DA) was obtained from Molecular Probes (Eugene, OR, USA), a subdivision of Invitrogen (Carlsbad, CA, USA). 2,2'-Azobis-(2-amidinopropane) dihydrochloride (AAPH) was obtained from Wako Chemicals (Richmond, VA, USA). Fluorescence readings of 96-well microtiter plates were performed by using a TECAN SpectraFluor plate reader (TECAN US, Durham, NC, USA).

\section{Serum testing for antioxidants, lipid peroxidation, and high-sensitivity CRP}

The CAP-e on serum was performed on all samples as described elsewhere. ${ }^{10,11}$ Serum samples were tested by using the thiobarbituric acid reactive substances assay from Cayman Chemical (Ann Arbor, MI, USA). In this assay, adducts formed by the reaction of malondialdehyde and thiobarbituric acid under high temperature and acid conditions can be measured colorimetrically at $535 \mathrm{~nm}$. Serum was tested for the concentration of CRP by enzyme-linked immunosorbent assay obtained from Alpha Diagnostics (San Antonio, TX, USA).

\section{Statistical analysis}

Statistical analysis involved comparisons between 2 mean values and was performed by using Microsoft Excel (Redmond, WA, USA). Statistical significance was tested by using the Student $t$-test, and a $P$ value less than .05 indicated a significant difference between 2 data sets. The means of grouped data were compared by using a 2-tailed unpaired (independent) $t$-test. The $P$ values in Table 2 reflect withinsubject analysis, in which the changes within the paired data sets for the same participant are analyzed before and after treatment (i.e., consumption) for each group.

\section{RESULTS}

\section{Rom}

In all study participants, the ROM at baseline was primarily reduced in the lumbar and lower-extremity areas. 
Table 2. Range of Motion Areas and Significance FOR IMPROVEMENTS

\begin{tabular}{llc}
\hline Anatomic area & \multicolumn{1}{c}{ Type of motion } & $\begin{array}{c}\text { P value for improvement } \\
\text { in ROM }\end{array}$ \\
\hline Cervical & Flexion/extension 12 weeks \\
Cervical & Rotation & $.2^{\mathrm{a}}$ \\
Thoracic & Flexion & \\
Thoracic & Rotation & \\
Lumbar & Flexion/extension & \\
Lumbar & Lateral left/right & $<.01^{\mathrm{b}}$ \\
Shoulder, left & Adduction/abduction & \\
Shoulder, left & Internal external rotation & \\
Shoulder, right & Adduction/abduction & \\
Shoulder, right & Internal external rotation & \\
Hip, left & Rotation & $<.04^{\mathrm{b}}$ \\
Hip, right & Rotation & $<.2^{\mathrm{b}}$ \\
Knee, left & Flexion/extension & $<.15$ \\
Knee, right & Flexion/extension & $<.06$ \\
\hline
\end{tabular}

${ }^{\text {a }} P$ values reflect "within-subject" analysis in which the changes are within the paired data sets for the after "treatment" (i.e., consumption).

${ }^{\mathrm{b}}$ The improvements in lumbar and hip rotation were seen at 8 weeks. The trends to improvement in cervical and knee range of motion were seen at 12 weeks, suggesting that it is possible that significance would have been reached in a study of longer duration.

Consumption of MonaVie Active resulted in an increase in lumbar and lower-extremity ROM as well as an improvement in overall generalized pain for the study group as a whole (Fig. 2). Comparison of the means of the grouped data showed that lumbar ROM improved over the entire 12 weeks $(P<.02)$. Lower-extremity ROM improved by 12 weeks $(P<.05)$

In an analysis of overall ROM for the 5 specific areas (cervical, thoracic, lumbar, and upper and lower extremities), the following picture emerged. Cervical rotation showed a trend to improvement at 12 weeks $(P<.2)$. Otherwise, the cervical (neck) area and the upper body (including shoulders) did not show major improvements during the 12-week study. However, lumbar and lower-body ROM improved-lumbar after 2-4 weeks and lower body (knees) by 8-12 weeks.

A second analysis involved identifying areas of reduced ROM, which focuses on the specific types of motion that are impaired for each person, such as bending the head backward or extending the right shoulder. We summarized the specific anatomic areas where a person at the start of the study had an ROM more than $25 \%$ lower than normal, and compared those to the number of anatomic areas with reduced ROM at study finish. The average number of areas of reduced ROM was $12.1 \pm 4.5$ at study start and $5.5 \pm 2.8$ at study finish. This indicates that after 12 weeks' consumption, on average $55 \%$ of the areas affected by reduced ROM had shown sufficient improvement that the areas no longer scored $25 \%$ or more outside normal ROM range. The data are shown in Table 1.

Data on muscle strength, including grip strength, did not show changes during this study (data not shown). This is probably due to the fact that approximately $90 \%$ of the study participants were within normal range at study start, and the hand/wrist was not a primary area of concern for any participant.

\section{Pain}

By nature, all pain assessment is essentially subjective. Whether using questionnaires, VAS, or mechanical assessment by algometry, the data collection requires the participant to report the perceived pain level as well as other aspects of quality of life and the ability to carry out activities associated with daily living. The tools we used were very different in nature, and the pain data collected during this study reflected different aspects of pain.

The level of a person's subjective perception of pain was assessed by using the VAS, a widely used and well-accepted research tool for pain studies. ${ }^{19}$ Its simplicity is very important for studies of this nature. Other scales use more graphically appealing questionnaires with smiley, sad, or crying faces. However, in our experience, these types of questionnaires are less suitable to research studies because people can memorize what score they chose at their last visit and will tend to score the same. We specifically chose the VAS because of its lack of reference points, which forces a person to think more about how to answer at each visit.

Data collected using the VAS and ADL questionnaire reflected aspects of pain directly relevant for a person's ability to function in daily life. Both data sets showed statistically significant improvements during the 12 weeks of study. The self-reported overall pain level, as scored on the VAS, was significantly reduced by 12 weeks of MonaVie Active consumption (Fig. $3 ; P<.01$ ).

The ADL questionnaire scores improved in most people. The overall ADLs were severely affected by pain for volunteers 05 and 09. Both these participants had severe health problems other than joint pain. When volunteers 05 and 09 were removed from analysis, the improvement in the ability to perform ADLs was also statistically significant $(P<.025)$.

In contrast, data collected by using the questionnaire describing the nature of the pain, as well as algometry, did not show significant changes (data not shown). The questionnaire contains a number of more qualitative questions, such as whether the pain is throbbing, piercing, or stabbing. Although pain, whether acute or chronic, is well understood to affect a person's daily activities and lessens a person's quality of life, the specific quality of the pain was not particularly useful in this instance. If pain is persistently present, it will probably affect daily activities, no matter the quality of the pain.

Pressure algometry applied along the 18 pressure points (found to be involved in chronic pain syndromes such as fibromyalgia), along with additional paraspinal points, was performed on all participants. Only 2 participants had symptoms associated with previously diagnosed fibromyalgia. Therefore, changes in most participants were expected to be minor. Individual analysis of the 2 people with fibromyalgia symptoms showed no improvement in pain tolerance at the specified points but revealed highly variable data throughout the study. 

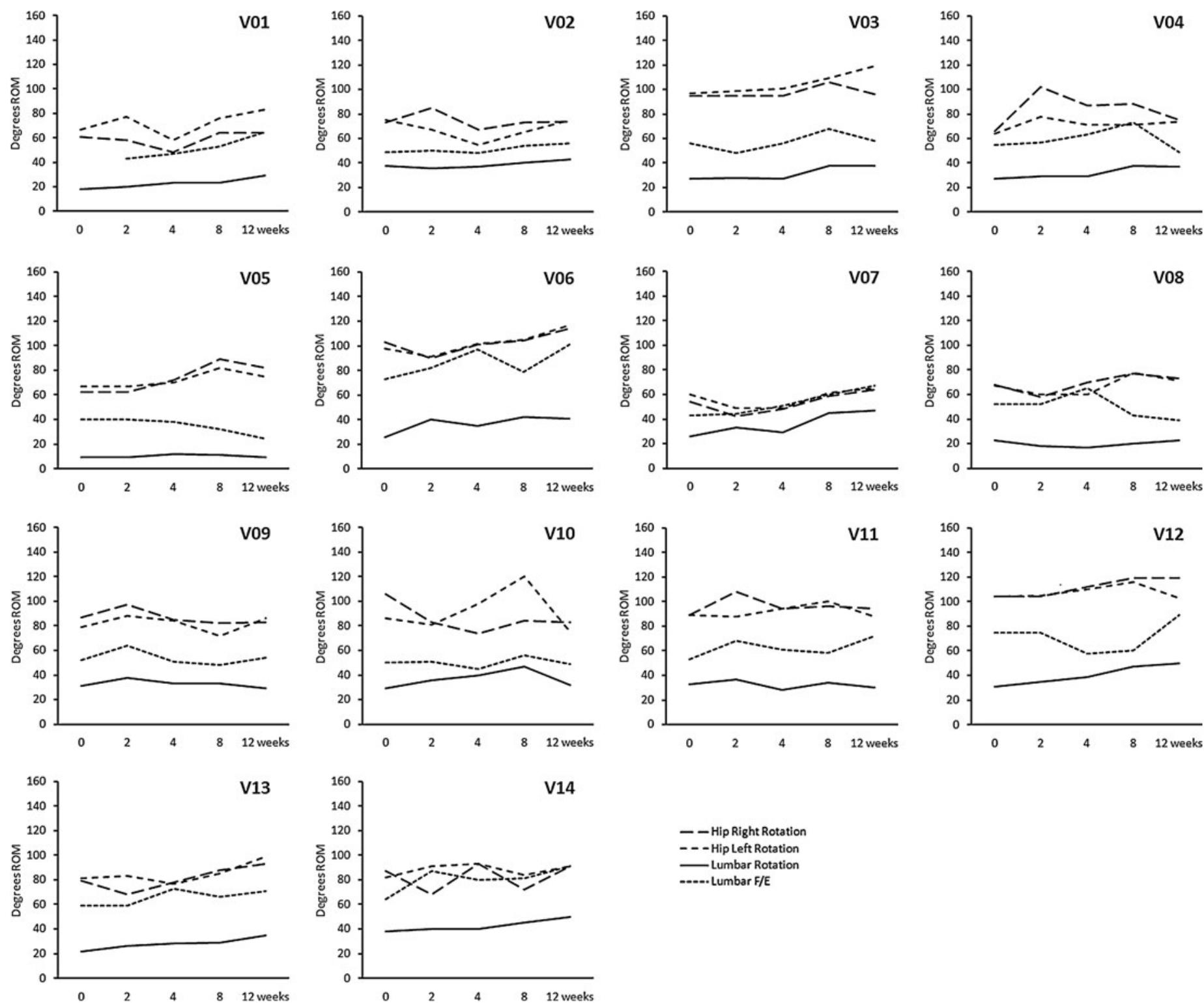

FIG 2. Individual range of motion (ROM) scores (in degrees) are shown for the 14 study participants over the 12 weeks of the study. ROM scores for hip right rotation (long dashes), hip left rotation (short dashes), lumbar rotation (solid line), and lumbar flexion/extension (F/E, dotted line) are shown. When these ROM measurements were evaluated by using a "within-subject" analysis, the overall improvements for lumbar F/E were significant at 2 weeks $(P<.05)$; the mild improvement remained constant for the rest of the study but did not maintain significance. The improvements to lumbar rotation reached statistical significance at 8 weeks and remained significant at 12 weeks $(P<.01)$. The improvements in hip ROM reached statistical significance for the left hip at 8 weeks and maintained significance through the 12 th week $(P<.04)$.

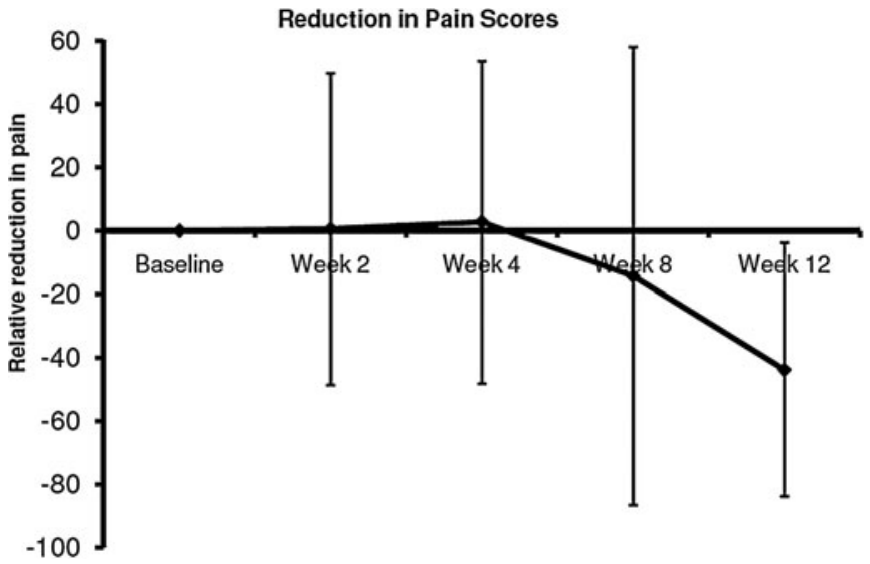

FIG 3. Changes in pain scores are shown over the 12-week study period. Some individuals reported improvements at 2-4 weeks, but the group average scores did not reach significance at that early point. The reduction in the group average pain scores reached statistical significance at 12 weeks of study $(P<.01)$. 
Table 3. Subjective Observations From Study Participants

\begin{tabular}{|c|c|c|c|c|}
\hline Volunteer & $\begin{array}{l}\text { Pain relief first } \\
\text { reported (wk) }\end{array}$ & $\begin{array}{c}\text { Increased } \\
\text { physical activity (wk) }\end{array}$ & $\begin{array}{l}\text { Medication } \\
\text { changes }(w k)\end{array}$ & $\begin{array}{c}\text { Specific changes } \\
\text { in medications, other observations }\end{array}$ \\
\hline 1 & 2 & 2 & & After 8 wk: reduced nocturnal enuresis and better sleep \\
\hline 2 & 4 & & & More energy, less asthma and seasonal allergies \\
\hline 3 & 2 & 2 & 8 & D: acetaminophen \\
\hline 4 & 2 & 2 & & \\
\hline \multicolumn{5}{|c|}{5} \\
\hline 6 & 4 & 8 & & \\
\hline 7 & & 12 & & More energy \\
\hline 8 & 12 & 12 & 8 & D: alprazolam; A: duloxetine \\
\hline 9 & 2 & & 8 & R: naproxen; D: prednisone; I: methotrexate; A: tramadol \\
\hline 10 & & 8 & & \\
\hline 11 & 4 & & & \\
\hline 12 & 4 & & & More energy, less muscle spasms \\
\hline \multicolumn{5}{|r|}{ 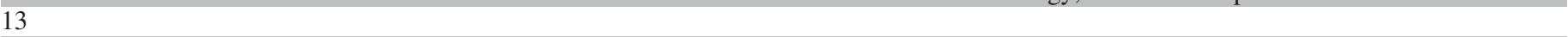 } \\
\hline 14 & 12 & & & \\
\hline
\end{tabular}

Participants were given the chance to discuss their experiences between clinic visits. Such observations as when pain relief was first noticed by the participants, when he or she could increase physical activity (e.g., exercise, chores), and medications changes were closely followed, along with other miscellaneous observations that may have been of interest.

A, added; D, discontinued; I, increased; R, reduced.

Emphasis was placed on allowing time for the study participants to visit with the research nurse at each clinic. Particularly in a study where the outcome measures focus on pain and inflammation, various stressors require careful monitoring. This includes exercise, mental stress, diet, supplement intake, and sleep. At each visit, the nurse asked each volunteer to fill out a tracking sheet for current health status, diet, medication, stimulants, stress level, and sleep. During the structured interview, many observations were tracked (Table 3). Several study participants experienced a rapid improvement, resulting in increased physical activity and ability to exercise. Eight of the 12 study participants reported reduced pain and an ability to increase their physical activities within the first 4 weeks, and several participants reported the improvements after 2 weeks. The participant with the most severe pain had ankylosing spondylitis and was being treated with methadone. This participant experienced improvements in terms of pain reduction at 8 and 12 weeks, even though the ROM measurements varied throughout the study.

Additional subjective observations were reported. Particularly, some study participants were able to alter their medications during the study, and some participants also experienced other apparently unrelated improvements. For example, an 84-year-old man experienced relief from nocturnal enuresis, which may suggest anti-inflammatory benefits in this participant.

\section{Serum antioxidant and inflammatory status}

Serum antioxidant status, as monitored by the CAP-e assay, was improved after 2 weeks of consumption of MonaVie Active and kept improving throughout the 12 weeks of the study (Fig. 4). The improvement in serum antioxidant protection status reached a high level of significance over the 12 weeks of study $(P<.01)$.

Lipid peroxidation did not change for the first 8 weeks. A mild decrease was observed at 12 weeks. One person (vol- unteer 08) had very high lipid peroxidation values for the last 2 visits and was removed from statistical analysis. Even with the removal of this outlier, only a mild trend was seen $(P<.16)$.

The inflammatory marker CRP was reduced after 12 weeks, but this finding did not reach statistical significance $(P<.22)$.

\section{Correlation between pain and antioxidant status but not CRP}

The data collected during this pilot study were primarily aimed at documenting health effects, particularly in terms of

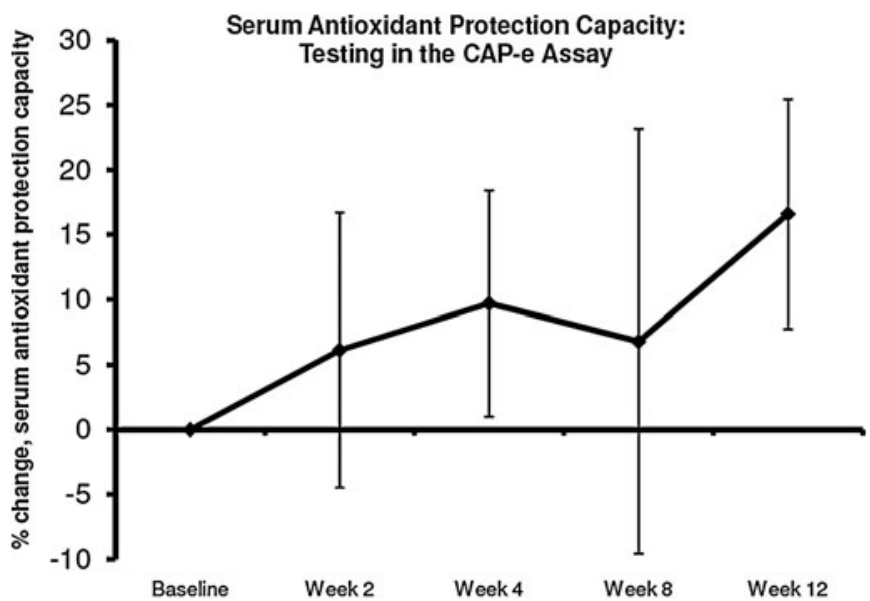

FIG 4. Improvement in serum antioxidant protection over the course of the 12-week study. Serum antioxidant protection status was measured by using the cell-based antioxidant protection in erythrocytes (CAP-e) bioassay; thus, the data reflect presence of serum antioxidants that are capable of entering into and protecting live cells. The improved status was seen at 2 weeks and continued to improve throughout the study, reaching a high level of significance at 12 weeks $(P<.01)$. 

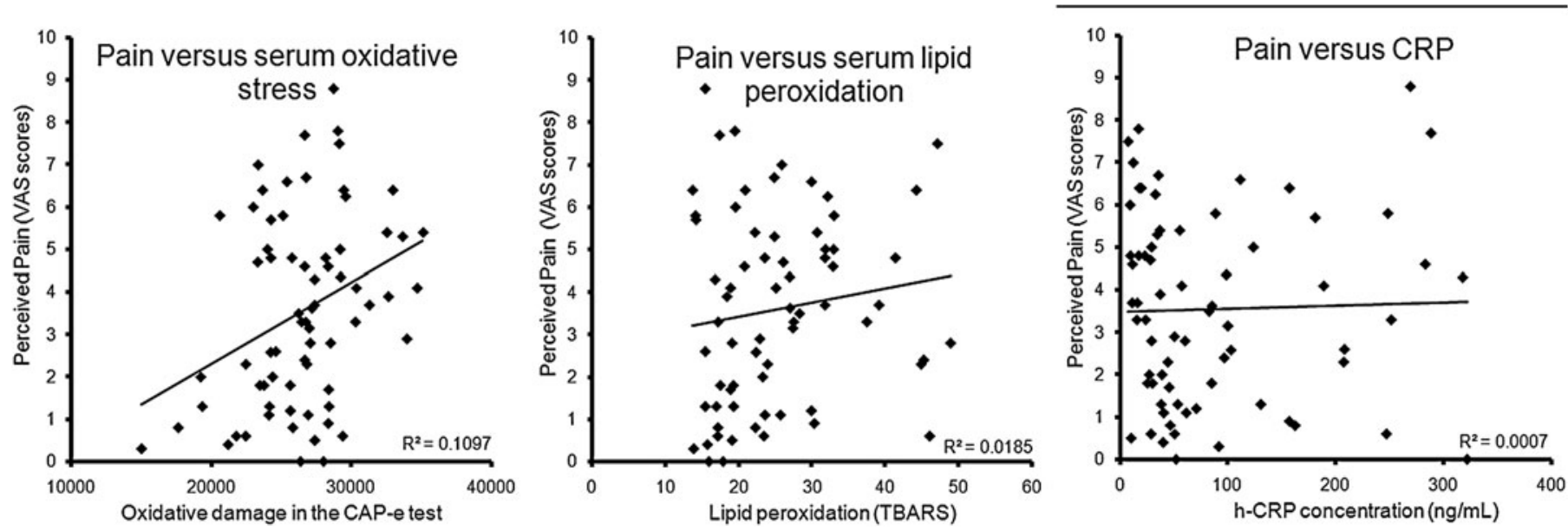

FIG 5. Pain scores in correlation to serum markers for oxidative stress and inflammation. A positive correlation was shown between higher perceived pain and higher levels of oxidative damage, as measured by the cell-based antioxidant protection in erythrocytes (CAP-e) bioassay $\left(\mathrm{R}^{2}=0.107\right)$. Only antioxidants that can enter and protect cells from oxidative damage are measured in this bioassay. ${ }^{11}$ Throughout the study, serum samples obtained after consumption of MonaVie Active were better able to protect cells from oxidative damage as visual analogue scale (VAS) scores decreased in the same individuals. Pain score versus lipid peroxidation data from serum samples showed no correlation between higher pain scores and higher lipid peroxidation $\left(R^{2}=0.0185\right)$. Higher pain score were not correlated to higher serum high-sensitivity $C$-reactive protein $(h-C R P)$ concentration $\left(\mathrm{R}^{2}=0.0007\right)$. TBARS, thiobarbituric acid reactive substances.

chronic inflammatory and pain problems. However, the data also lend themselves to a cross-sectional analysis to examine correlations between pain levels and the 3 different blood tests performed.

The ROM improvement, pain reduction, and improved ADLs showed, as expected, a good correlation. Therefore, pain was selected as the key variable for examining which of the serum tests showed the best correlation with how people were performing in their daily life.

Of note, the correlation between pain level and poor antioxidant status was strongest (Fig. 5). There was a moderate correlation between pain level and lipid peroxidation status. There was no correlation between pain level and high sensitivity CRP.

\section{DISCUSSION}

Healthy aging is associated with full cognitive function and the absence of pain. Some forms of age-related cognitive impairment as well as chronic pain conditions have been linked to lower levels of endorphins in spinal fluid. This finding is in turn associated with a reduction in the pain threshold. For some people this downward spiral lasts for many months or years, regardless of whether the problem resulted from an injury, infection, or neurologic damage. It is challenging to reverse the cycle of stimuli that are maintaining problems associated with chronic pain. Furthermore, the cognitive impairment seen with chronic pain is an often underappreciated issue with deep effects on a person's ability to function physically, socially, and emotionally.

It is of growing interest to identify natural products by using a multifaceted approach to healthy aging, neuroprotection, and pain management. This includes improved anti-oxidant status, reduction in chronic inflammation, sup- port of cognitive function, and improved energy production. Several foods and dietary constituents are associated with reduced pain perception in animals and humans. From the perspective of the data presented in this paper, it is important that foods rich in a broad array of polyphenols, such as from tart cherries, ${ }^{5-7}$ wild blueberries, ${ }^{20}$ Concord grapes,${ }^{21}$ and green tea, ${ }^{22}$ have been found to have anti-nociceptive effects. Isolated compounds studied for their anti-nociceptive effects include resveratrol, ${ }^{23,24}$ curcumin, ${ }^{25}$ and clorogenic acid. ${ }^{26}$ The most direct mechanism of action proposed for explaining this effect is the COX-2 inhibition by such compounds; however, other mechanisms probably contribute as well. Direct and indirect effects on nitric oxide and vascular blood perfusion, increased oxygen delivery to brain and other tissue, ${ }^{27}$ and direct effects on cognitive function and production of endorphins may contribute to an overall effect.

Açai contains a range of polyphenols that protect cellular oxidative damage in vitro and provide anti-inflammatory signaling leading to reduced production of free radicals by inflammatory cells. ${ }^{12}$ The current study was performed as an extension of our previous in vitro studies of açai and was based on pilot data from a human randomized, doubleblinded, placebo-controlled crossover study on acute effects on antioxidant uptake and inflammatory markers in a healthy population. The data presented here were generated from a consumption study involving 14 people with a broad variety of chronic pain issues affecting their ROM and ability to perform ADLs.

The perceived pain changed significantly over the course of the study. The more objective ROM measures were affected by a combination of perceived pain and mechanical performance as affected by inflammation. Some people experienced improvements as early as $2-4$ weeks. This improvement led to increased physical activity; some of 
these patients subsequently developed secondary pain problems. Thus, reduced pain and improved ROM led to increased physical activity, followed by transient soreness as muscles were rebuilding. These fluctuations, as can be seen from Figure 2, had resolved by 12 weeks.

Lumbar improvements were seen earlier than improvements in lower extremities. We believe this finding occurred because the lower extremities are under the most physical stress and therefore require more time to register improvements. The cervical and upper extremities did not show significant improvements; however, this result was expected because these were not the primary areas of concern for most participants. Areas that did not have problems at study start should not be expected to improve, whereas areas that had limited ROM at study start might be expected to show signs of change. Therefore, a second round of analysis focused on the anatomic areas that showed limitations at study start. Analysis of only the subset of ROM data that were lower than the normal range at study start showed statistically significant improvements as early as the second week; this subset of patients continued to improve throughout the study.

One of the most interesting observations emerging from the analysis of the overall data was the correlation between elevated pain levels and poor antioxidant status. The antioxidant status, as measured by the CAP-e, showed the best correlation with improvements in physical health. Antioxidants as a whole have been scrutinized as of late, mainly because of a meta-analysis of studies involving such vitamins as ascorbic acid and vitamin E-tocopherols. It is crucial to separate studies of single antioxidant vitamins from studies involving foods containing complex arrays of polyphenols. The data presented here show a correlation between pain levels and antioxidant status but only a poor or no correlation to lipid peroxidation and CRP. We hope that these data will encourage other studies on complex natural products that contain a broader spectrum of polyphenol antioxidants, in terms of effects of consumption on complex issues such as pain perception and cognitive function.

The association between chronic pain and cognitive function, and the emerging evidence that polyphenols have neuroprotective and cognitive effects, deserves further attention. The emerging recognition of the fact that intake of high-polyphenol foods, such as berries, fruits, and nuts, provides a broad-spectrum support of neurologic and vascular functions may indeed change our management of many chronic health conditions.

\section{ACKNOWLEDGMENTS}

The study was commissioned by AIBMR Life Sciences and performed at NIS Labs, an independent contract research laboratory. The study was sponsored by MonaVie LLC.

\section{AUTHOR DISCLOSURE STATEMENT}

A.G.S. currently serves as an uncompensated member on the scientific advisory board of the sponsor, MonaVie LLC.

\section{REFERENCES}

1. Fries J: Exercise and the health of the elderly. Am J Geriatr Cardiol 1997;6:24-32.

2. Petrella JK, Elaine Cress M. Daily ambulation activity and task performance in community-dwelling older adults aged 63-71 years with preclinical disability. J Gerontol Series A 2004;59: M264-M267.

3. Rogers MA, Hagberg JM, Martin WH, Ehsani AA, Holloszy JO: Decline in VO2max with aging in master athletes and sedentary men. J Appl Physiol 1990;68:2195-2199.

4. Tall JM, Raja SN: Dietary constituents as novel therapies for pain. Clin J Pain 2004;20:19-26.

5. Tall JM, Seeram NP, Zhao C, et al.: Tart cherry anthocyanins suppress inflammation-induced pain behavior in rat. Behav Brain Res 2004;153:181-188.

6. Kuehl KS, Perrier ET, Elliot DL, Chesnutt JC: Efficacy of tart cherry juice in reducing muscle pain during running: a randomized controlled trial. J Int Soc Sports Nutr 2010;7:17.

7. Connolly DA, McHugh MP, Padilla-Zakour OI, Carlson L, Sayers SP: Efficacy of a tart cherry juice blend in preventing the symptoms of muscle damage. Br J Sports Med 2006;40:679-683; discussion 683.

8. Schauss AG, Wu X, Prior RL, et al.: Phytochemical and nutrient composition of the freeze-dried Amazonian palm berry, Euterpe oleraceae Mart. (Acai). J Agricul Food Chem 2006;54:85988603.

9. Schauss AG, Wu X, Prior RL, Ou B, et al.: Antioxidant capacity and other bioactivities of the freeze-dried Amazonian palm berry, Euterpe oleraceae Mart. (açai). J Agricul Food Chem 2006; 54:8604-8610.

10. Jensen GS, Wu X, Patterson KM, et al.: In vitro and in vivo antioxidant and anti-inflammatory capacities of an antioxidantrich fruit and berry juice blend. Results of a pilot and randomized, double-blind, placebo-controlled, crossover study. J Agric Food Chem 2008;56:8326-8333.

11. Honzel D, Carter SG, Redman KA, et al.: Comparison of chemical and cell-based antioxidant methods for evaluation of foods and natural products: generating multifaceted data by parallel testing using erythrocytes and polymorphonuclear cells. J Agric Food Chem 2008;56:8319-8325.

12. Kang J, Li Z, Wu T, et al.: Antioxidant capacities and antiinflammatory effects of flavonoid compounds from açai pulp (Euterpe oleracea Mart.). Food Chem 2010;122:610-617.

13. Schauss AG: Açai (Euterpe oleracea Mart.): a macro and nutrient rich palm fruit from the Amazon rain forest with demonstrated bioactivities in vitro and in vivo. In: Bioactive Foods in Promoting Health:Fruits and Vegetables. Watson RR, Preedy V, eds.. Oxforx, Academic Press, 2010, pp. 479-490.

14. Sun X, Seeberger J, Albericol T, Schauss AG, Zou S: Açai palm fruit (Euterpe oleracea Mart.) pulp improves survival of flies on a high fat diet. Exp Gerontol 2010;45:243-251.

15. Kirby K, Hu J, Hilliker AJ, Phillips JP: RNA interference-mediated silencing of Sod2 in Drosophila leads to early adult-onset mortality and elevated endogenous oxidative stress. Proc Natl Acad Sci U S A 2002;99:16162-16167.

16. Phillips JP, Campbell SD, Michaud D, Charbonneau M, Hilliker AJ: Null mutation of copper/zinc superoxide dismutase in Drosophila confers hypersensitivity to paraquat and reduced longevity. Proc Natl Acad Sci U S A 1989;86:2761-2765. 
17. Bogoyevitch MA, Ngoel KRW, Zhao TT, Yeap YYC, Ng DCH: $\mathrm{C}$-jun N-terminal kinase (JNK) signaling: recent advances and challenges. Biochim Biophys Acta (BBA) Proteins Proteomics 2010;1804:463-475.

18. Schauss AG, Clewell A, Balogh L, et al.: Safety evaluation of an açai-fortified fruit and berry functional juice beverage (Monavie Active). Toxicology 2010;278:46-54.

19. Huskisson EC: Measurement of pain. J Rheumatol 1982;9:768769.

20. Papandreou MA, Dimakopoulou A, Linardaki ZI, et al.: Effect of a polyphenol-rich wild blueberry extract on cognitive performance of mice, brain antioxidant markers and acetylcholinesterase activity. Behav Brain Res 2009;198:352-358.

21. Krikorian R, Nash TA, Shidler MD, Shukitt-Hale B, Joseph JA: Concord grape juice supplementation improves memory function in older adults with mild cognitive impairment. $\mathrm{Br} J \mathrm{Nutr}$ 2010;103:730-734.

22. Kim HK, Kim M, Kim S, Kim M, Chung JH: Effects of green tea polyphenol on cognitive and acetylcholinesterase activities. Biosci Biotechnol Biochem 2004;68:1977-1979.
23. Abraham J, Johnson RW: Consuming a diet supplemented with resveratrol reduced infection-related neuroinflammation and deficits in working memory in aged mice. Rejuvenation Res 2009;12:445-453.

24. Pham-Marcou TA, Beloeil H, Sun X, et al:: Antinociceptive effect of resveratrol in carrageenan-evoked hyperalgesia in rats: prolonged effect related to COX-2 expression impairment. Pain 2008;140:274-283.

25. Ataie A, Sabetkasaei M, Haghparast A, Moghaddam AH, Kazeminejad B: Neuroprotective effects of the polyphenolic antioxidant agent, Curcumin, against homocysteine-induced cognitive impairment and oxidative stress in the rat. Pharmacol Biochem Behav 2010;96:378-385.

26. dos Santos MD, Almeida MC, Lopes NP, de Souza GE: Evaluation of the anti-inflammatory, analgesic and antipyretic activities of the natural polyphenol chlorogenic acid. Biol Pharm Bull 2006;29:2236-2240.

27. Kennedy DO, Wightman EL, Reay JL, et al.: Effects of resveratrol on cerebral blood flow variables and cognitive performance in humans: a double-blind, placebo-controlled, crossover investigation. Am J Clin Nutr 2010;91:1590-1597. 